\title{
Web-Based Interventions Alone or Supplemented with Peer-Led Support or Professional Email Counseling for Weight Loss and Weight Maintenance in Women from Rural Communities: Results of a Clinical Trial
}

\author{
Patricia A. Hageman, ${ }^{1}$ Carol H. Pullen, ${ }^{2}$ Melody Hertzog, ${ }^{3}$ Bunny Pozehl, ${ }^{3}$ \\ Christine Eisenhauer, ${ }^{4}$ and Linda S. Boeckner ${ }^{5}$ \\ ${ }^{1}$ Physical Therapy Education, College of Allied Health Professions, University of Nebraska Medical Center, \\ 984420 Nebraska Medical Center, Omaha, NE 68198-4420, USA \\ ${ }^{2}$ College of Nursing, University of Nebraska Medical Center, 985330 Nebraska Medical Center, Omaha, NE 68198-5330, USA \\ ${ }^{3}$ College of Nursing, University of Nebraska Medical Center, Lincoln Division, 1230 “O” Street, Suite 131, Lincoln, NE 68588-0220, USA \\ ${ }^{4}$ College of Nursing, University of Nebraska Medical Center, Northern Division, 801 E. Benjamin Avenue, Norfolk, \\ NE 68701-0469, USA \\ ${ }^{5}$ Nutrition and Health Sciences, College of Education and Health Sciences, University of Nebraska-Lincoln, 110 Leverton, \\ Lincoln, NE 68583-0806, USA
}

Correspondence should be addressed to Patricia A. Hageman; phageman@unmc.edu

Received 23 November 2016; Revised 4 March 2017; Accepted 15 March 2017; Published 5 April 2017

Academic Editor: Aron Weller

Copyright (c) 2017 Patricia A. Hageman et al. This is an open access article distributed under the Creative Commons Attribution License, which permits unrestricted use, distribution, and reproduction in any medium, provided the original work is properly cited.

\begin{abstract}
Objective. This trial compared the effectiveness of a web-based only (WO) intervention with web-based supplemented by peerled discussion (WD) or professional email counseling (WE) across 3 phases to achieve weight loss and weight maintenance in women from underserved rural communities. Methods. 301 women (BMI of $28-45 \mathrm{~kg} / \mathrm{m}^{2}$ ) randomly assigned to groups participated in guided weight loss (baseline to 6 months), guided weight loss and maintenance (6 to 18 months), and self-managed weight maintenance (18 to 30 months). Results. Retention was $88.7 \%, 76.5 \%$, and $71.8 \%$ at 6,18 , and 30 months, respectively. Intent-to-treat analyses demonstrated no group differences in change in weight within any phases. At 6 months, observed mean (SD) weight loss was $5.1(6.0) \mathrm{kg}$ in $\mathrm{WO}, 4.1(5.6) \mathrm{kg}$ in WD, and $6.0(6.3) \mathrm{kg}$ in WE, with $42 \%, 38 \%$, and 51\%, respectively, meeting $\geq 5 \%$ weight loss. These proportions dropped by a third after phase 2 with no further change during phase 3. Conclusion. Web-based interventions assisted women from rural communities in achieving 6-month weight loss, with weight regain by half at 30 months. No group differences were potentially due to the robust nature of the web-based intervention. Trial Registration. This trial is registered with ClinicalTrials.gov NCT01307644.
\end{abstract}

\section{Introduction}

Women from rural communities represent one of the largest medically underserved populations in the United States, with high rates of overweight and obesity, physical inactivity, and poor diets placing these women at high risk for developing chronic diseases, such as diabetes, heart disease, and stroke [1-3]. Behavioral approaches for lifestyle modification are recommended for treating overweight and obesity, yet women from rural communities have limited access to preventive services, such as counseling in diet and activity [3-5]. The 2011 Strategic Plan for the National Institutes of Health Obesity Research highlighted the need for designing and testing innovative lifestyle interventions to reach underserved rural populations, who face unique barriers to healthy behaviors [6].

The number of web-based lifestyle behavior change interventions targeting weight loss and weight maintenance has 
dramatically increased over the past 10 years, in part due to increased web accessibility and its potential for being a cost-effective method to address behavior change in hardto-reach populations [7-9]. The majority of studies of both weight loss and weight maintenance focus on web-based delivery as a supplement to face-to-face counseling or as an alternative to face-to-face counseling $[7,8]$. Web-based interventions result in smaller weight losses and lower levels of weight maintenance compared to in-person interventions, though small weight changes may be clinically relevant on a public health level as compared to no interventions [7, 8]. Few studies have investigated interventions delivered solely via web, or web-based with additive elements, and/or of a duration greater than four to six months $[7,8]$.

The challenge with any lifestyle modification intervention is to engage the participants to achieve $5 \%$ to $10 \%$ of body weight loss, the percentage loss associated with health benefits [10, 11]. Keeping participants engaged over longer periods of time, as needed for weight loss maintenance, presents greater issues and challenges, as weight regain occurs consistently within six to nine months of initial weight loss for most individuals [12, 13]. Maintaining engagement in purely web-based weight loss and weight maintenance interventions may be particularly challenging, though reviews of technology-based studies identified strategies that may be effective such as self-monitoring, goal setting, theorybased behavior change messaging, social support, and skill building to prevent weight regain [13-16]. In multiple studies of web-based weight loss and weight maintenance, greater adherence or intervention dosage, as measured by attendance in online chats or login frequency, was associated with weight change [17-20]. In face-to-face lifestyle weight loss interventions, additional support from peers or professionals enhanced participant engagement, adherence, and weight outcomes [13, 16, 21]. Providing additional support such as email counseling and peer-led discussion in web-based studies could potentially boost participant engagement and improve weight loss outcomes $[9,16,21,22]$.

Web-based weight loss and/or weight maintenance studies that augmented a web-based only group with individualized feedback in the form of email counseling, e-coaching, or automated computer feedback had varying results, possibly due to differing intervention durations (three to 12 months), though participants receiving individual feedback at three months were observed to lose more weight than those receiving no additional feedback, and the effect was slightly greater for email counseling [7, 17-20, 23]. Outcomes of other webbased studies using supplemental support via professional email counseling showed promising yet inconclusive results [24-26].

Studies of the effectiveness of web-based peer-led support groups varied in how the support was provided, as some provided peer support by health professionals, and others used multicomponent interventions where the web-based peer component effects were not separated for analysis $[8,18,20,24]$. In our three-month pilot study of women aged 50-69 from rural communities, we found that access to a web-based intervention supplemented with a peer-led asynchronous support group doubled the engagement with the web intervention and enhanced weight loss compared to a web-based only group [27].

Few studies have compared purely web-based weight loss and weight maintenance interventions to those with enhanced features or have targeted women from rural communities, considered a designated priority population for study due to documented health disparities [28]. This study is unique, as all three web-based intervention groups receive three specific intervention phases progressing from intensive guided weight loss tapering to guided weight maintenance and finally self-management of weight maintenance, and the study offers longer weight maintenance interventions, as recommended by the Institute of Medicine for optimal longterm outcomes $[29,30]$.

The conceptual framework of this study was based upon Pender's Health Promotion Model (HPM), which is founded on social cognitive theory, in order to explain and modify the adoption and maintenance of healthy eating and activity behaviors necessary to achieve weight loss and weight maintenance [31]. The intervention messaging for behavior change focused on perceived benefits of action, perceived barriers to action, self-efficacy, and interpersonal influences of family and friends for action, all of which are behaviorspecific cognitions from the HPM. The interventions also focused on the commitment to a plan of action through goal setting.

The primary aim of the study was to compare the effectiveness of a web-based only (WO) intervention with webbased interventions supplemented with peer-led discussion support group (WD) or professional email counseling (WE) for achieving change in primary outcomes of body weight and waist circumference and secondary outcomes of attaining criterion weight loss targets (5\% and $10 \%$ weight loss) by phase: phase 1 for guided weight loss (baseline to 6 months), phase 2 for guided continuing weight loss and maintenance (6 to 18 months), and phase 3 for self-managed weight maintenance (18 to 30 months).

The secondary aim was to compare the three groups on improvement in primary outcomes of healthy eating and activity (Kcal intake, weekly minutes of moderate or greater intensity activity, blood pressure, and lipids), as well as achieving secondary outcomes of eating and activity at recommended criterion targets $(20-35 \%$ of daily fat kcal intake and 150 weekly minutes or greater of moderate or greater physical activity) by the end of each of the three phases.

Based upon the literature and our pilot work, we hypothesized that each of the intervention groups receiving supplemental elements, either WD or WE, would have better outcomes than the WO group. The evidence in the literature was insufficient to hypothesize the relative effectiveness of the peer-led discussion support group compared to the professional email counseling.

\section{Methods}

Ethical approval for this study was received through the Institutional Review Board of the University of Nebraska Medical 
Center (approval number: 23710-FB). Written informed consent was obtained from all participants, following the standardized protocol prior to their enrollment. The study was conducted between June 2011 and December 2014, with recruitment, enrollment, and baseline assessments occurring over one year (June 2011 to May 2012). Eligible women were randomly allocated to 1 of 3 parallel intervention groups (1:1:1 ratio), with each group receiving three intervention phases over a 30-month period [29].

2.1. Participants. A total of 301 women, aged 40-69, who met rural status as defined by the Rural-Urban Commuting Area (RUCA) codes [32], which classifies rural based upon population density and population work commuting patterns, were recruited from northeastern Nebraska in the USA. The study specifically targeted women, as opposed to both genders, in response to longitudinal data showing women having a disproportionately higher rate of late-life disability than men, which might be reduced through genderappropriate preventive interventions earlier in life [33].

Women were eligible for the study if they had a BMI of $28-45 \mathrm{~kg} / \mathrm{m}^{2}$ and were not taking medications that affected weight loss or weight gain, as verified by research personnel. Other inclusion criteria included ability to speak and read English, communicate over the phone, and use a computer including email features with minimal assistance. They also needed to have access to the Internet and a DVD player and be willing to drive up to 70 miles each way to our centrally located research office at a community college for all required assessments. Eligible women were included if they answered "no" to all questions on the Physical Activity Readiness Questionnaire (PAR-Q) or obtained medical clearance from their physician to participate [34]. Major exclusion criteria were as follows: having a diagnosis of type 1 diabetes or having a diagnosis of type 2 diabetes and requiring insulin, having $10 \%$ or greater weight loss in the last six months, being currently enrolled in a weight loss management program or another research study, participating in current cancer treatment, or having physical or medical restrictions that would preclude following recommendations for moderate activity and healthy eating.

2.2. Trial Conduct. A project recruiter was hired from the region to assist in recruiting women through local advertising and direct mailings, with special efforts to recruit persons from underrepresented racial/ethnic backgrounds and/or individuals with lower socioeconomic status through local community contacts. Women expressing interest in the study received a phone follow-up screening interview conducted by the recruiter or other study personnel to verify that all eligibility requirements were met.

Research nurses completed the informed consent process with eligible women at the assessment site. Baseline assessments were conducted over two visits scheduled within three weeks. At baseline visit one, nurses provided each woman with a pedometer [Omron HJ-112 GoSmart, Omron Healthcare, Inc., 1925 W Field Ct, Lake Forest, IL 60045] and an accelerometer, with instructions for their use. Nurses dispensed book of food counts [35] and a web user's guide to women (designed to assist women in accessing and using the intervention website). At completion of the first baseline visit, nurses delivered a sealed confidential envelope to each woman that contained an identification number and a password for logging into the intervention website and advised women to keep their login information materials confidential. Women were informed of a "practice period" between baseline visits one and two during which they would be asked to access and become acquainted with their intervention website by logging weight, food intake, and pedometer steps. The intent of the practice period was to further inform the women about the nature of the project, assess their willingness to self-monitor, and verify computer and web literacy. If the women did not participate in the practice period as noted by the technical support team, they were dropped from the study. At the second baseline visit, women repeated selected measures for reliability purposes (e.g., blood pressure) and performed any remaining assessments not completed during baseline visit one.

2.2.1. Randomization and Blinding. A randomization schedule was created by the project statistician using online software to generate sequences of pseudorandom numbers (http://www.randomizer.org/form.htm). To keep accrual relatively even during rolling enrollment, a random ordering of block sizes 12,15, and 18 was used. The project statistician did not have any contact with the women during the trial. Upon completion of baseline assessment visit two, each woman received an electronic notice on her intervention web account of her group assignment. The women were instructed to not share this with others, including the research nurses who conducted the assessments, who were blinded to webintervention content as well.

\section{Interventions}

3.1. Web Intervention for All Groups. Women in all three groups had access to the same content on the intervention website, except that two groups received either a supplemental peer-led discussion blog or professional email counseling. All women received a behavior change lifestyle plan on the website, consisting of eating and activity recommendations based upon the 2010 Dietary Guidelines for Americans [36], the 2008 Physical Activity Guidelines for Americans [37], and Healthy People 2010 [38]. Dietary recommendations included reduction in caloric intake (range 1,200 to 1,600 Kcal), an average daily fat intake of $20-35 \%$, and a focus on nutrient-dense food and beverages [36, 38]. For physical activity, women were encouraged to achieve a criterion activity level of $\geq 150$ minutes weekly of moderate or greater activity, and if this was achieved, they were encouraged to increase their activity to 60 to 90 daily minutes of moderate or greater physical activity as recommended for weight loss maintenance $[39,40]$.

Table 1 summarizes the interventions delivered to each of the three groups. Intervention details are included in the protocol paper [29], and specific web content denoted by 


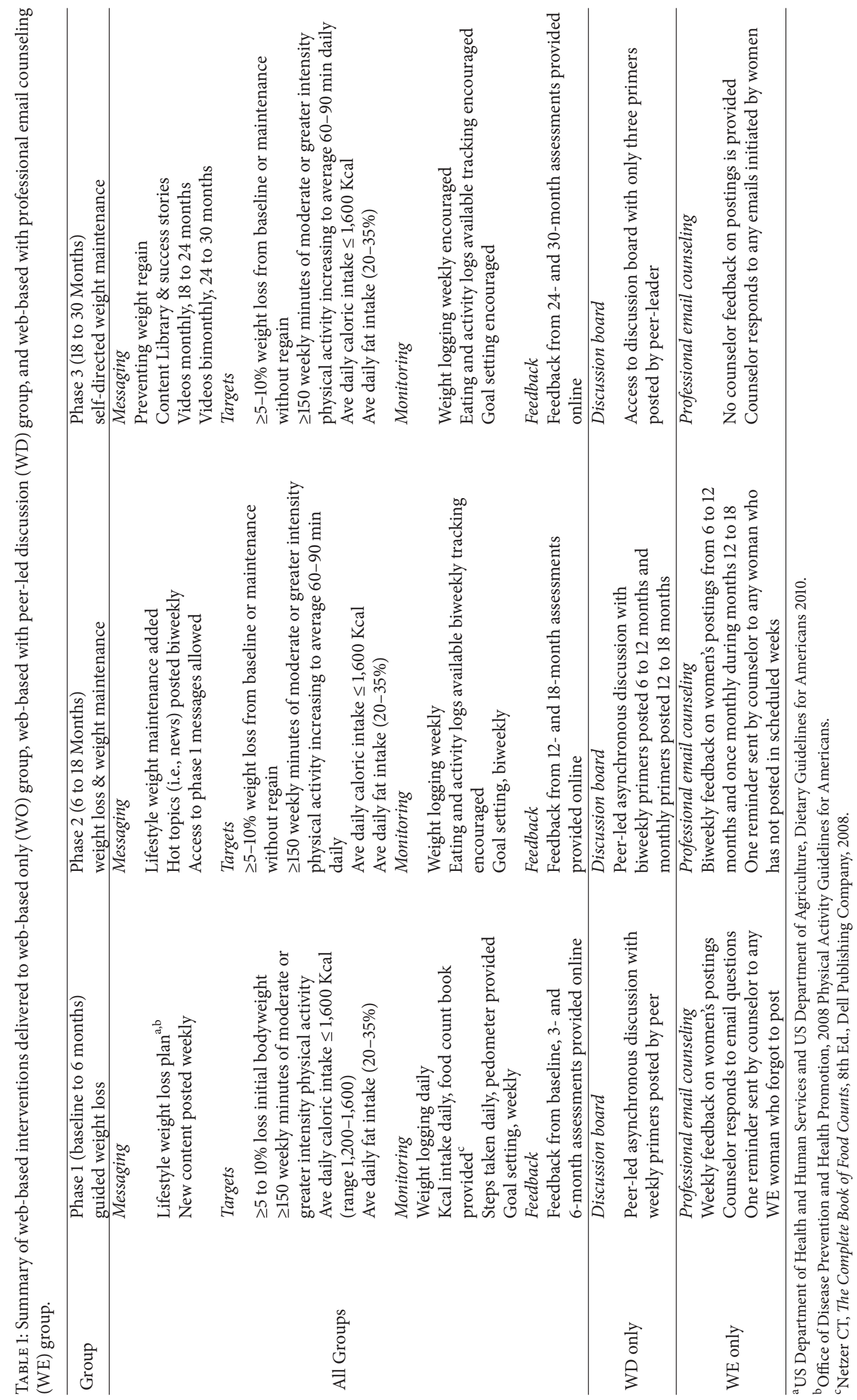


theoretical construct for each time point of delivery by phase is reported on the clinical trial website (http://unmc.edu/ alliedhealth/research/ww4w). Sample website screen views are included in additional files [see Supplemental Illustration 1 in Supplementary Material available online at https://doi .org/10.1155/2017/1602627]. Women had access to technology support throughout the entire 30-month study from the project technologist.

3.2. Peer-Led Discussion Group. Women in the WD group could view an extra tab on the intervention home page labeled "Discussion." This asynchronous discussion board was managed by a peer-leader, trained by the project investigators, who was responsible for posting new theme-based messages, called primers, on a predetermined schedule by phase, as noted in Table 1. When posting message responses, each participant had a unique and nonpersonalized identifier. A detailed listing of the content of discussion board posts by phase is located on the trial website (http://unmc.edu/ alliedhealth/research/ww4w).

3.3. Professional Email Counseling. Women in the WE group received emails from a registered dietitian whose identity was masked. The email counselor was responsible for reviewing the WE women's web-based logs of weight, eating, activity, and goal setting and sending an email with feedback using a structured process [41] using a frequency which was consistent with the WD group "primers" during phases 1 and 2 (see Table 1). The WE participants were allowed, but not required, to respond to any email they received from the professional counselor. During phase 3, the women were notified that they could email the professional counselor at any time with questions; however, the email counselor only responded to participant-initiated emails.

3.4. Intervention Unexpected Events. The only major unexpected event that may have influenced intervention delivery was a technical issue that occurred on April 21, 2012, nearing the May 2012 completion date for enrollment, when the server which housed the study's website upgraded its security, making computers using one of the default settings in Internet Explorer (TLS 1.0) unable to read the website. The problem was discovered on April 22, and the technical support team made contact with women to rectify the issue with verification that all women were able to $\log$ in by May 11, 2012.

3.5. Outcomes. Table 2 outlines the primary and secondary outcomes for the primary aim (weight) and secondary aim (eating and activity), with a listing of the measurement instruments used and frequency of the assessments. The details of the assessment and measurements have been published previously [29], and a brief summary is provided below. Women completed surveys of general demographic information and health history. Measures of height and weight were assessed using the Tanita scale [TBF-215, Tanita Corporation of American, Inc., $2625 \mathrm{~S}$ Clearbrook Dr., Arlington Heights, IL 6005-9824]. BMI was calculated as weight in kilograms divided by height in meters squared. Waist circumference was measured using a snug tape parallel to the floor but held without skin compression around the abdomen located at the level of the iliac crest at the end of expiration, with the average of two trials recorded [42].

The key behavioral measures related to eating (i.e., kcal intake daily, percent daily calories from fat) were assessed using the web version of the 1998 Block Health Habit and History Questionnaire, which asked for the frequency of consumption of particular food items during the last three months [43]. Prior research has shown this instrument to demonstrate high reliability, validity, and sensitivity to change [44-46].

Standardized methods were followed to assess women's estimated daily minutes of moderate or greater physical activity over seven days using the lightweight triaxial Actigraph Accelerometer (Model GT3X, Pensacola, FL), shown to have established reliability and validity [47-49]. Women were asked to wear the Actigraph on the dominant hip attached to an elastic waist band for 24 hours a day, except when showering or swimming, over seven days. Adherence to the wear guidelines was high at all times. Mean days of wear ranged from $6.8(\mathrm{SD}=0.6)$ at baseline to 6.6 days $(\mathrm{SD}=$ 1.2 ) at 30 months, with the percentage of women wearing the Actigraph on six or seven days exceeding $94 \%$ at all times.

Self-reported weekly minutes of moderate or greater physical activity were assessed using the Behavioral Risk Factor Surveillance System (BRFSS) Physical Activity module consisting of seven items that have been shown to have acceptable reliability and validity [50]. Following the methods used by the Women's Injury Study, the BRFSS questions were administered to our women to estimate the weekly minutes of moderate or greater physical activity [51].

Biomarkers affected by eating and activity included blood pressure and blood lipids. Following 5 minutes of quiet sitting, blood pressure was measured using standardized methods [52]. Women were asked to fast for 12 hours prior to assessments to determine fasting blood lipids that required a blood draw by the research nurses and were processed using a standardized protocol [53].

Other data, including women's perceptions of behavioral determinants and process evaluations via surveys and focus groups, were collected as described previously [29]. These results will be reported in a future paper.

3.6. Website Usage. Tracking of the women's access to and use of the intervention website occurred by documenting the number of logins, including date and time, and their "clicks" on various features within the website, including clicks on the discussion board items for those enrolled in the WD group. The proxy for dosage was the count of women's logins by time points of new content, regardless of whether they accessed one or more of the web features or whether they logged in one or multiple times during a given week. For example, a woman who logged in on each of the 26 weeks of new web content during phase 1 was considered to have $100 \%$ dosage. Dosage of use of the discussion board was defined similarly, noting women's clicks specific to the discussion board site by time 


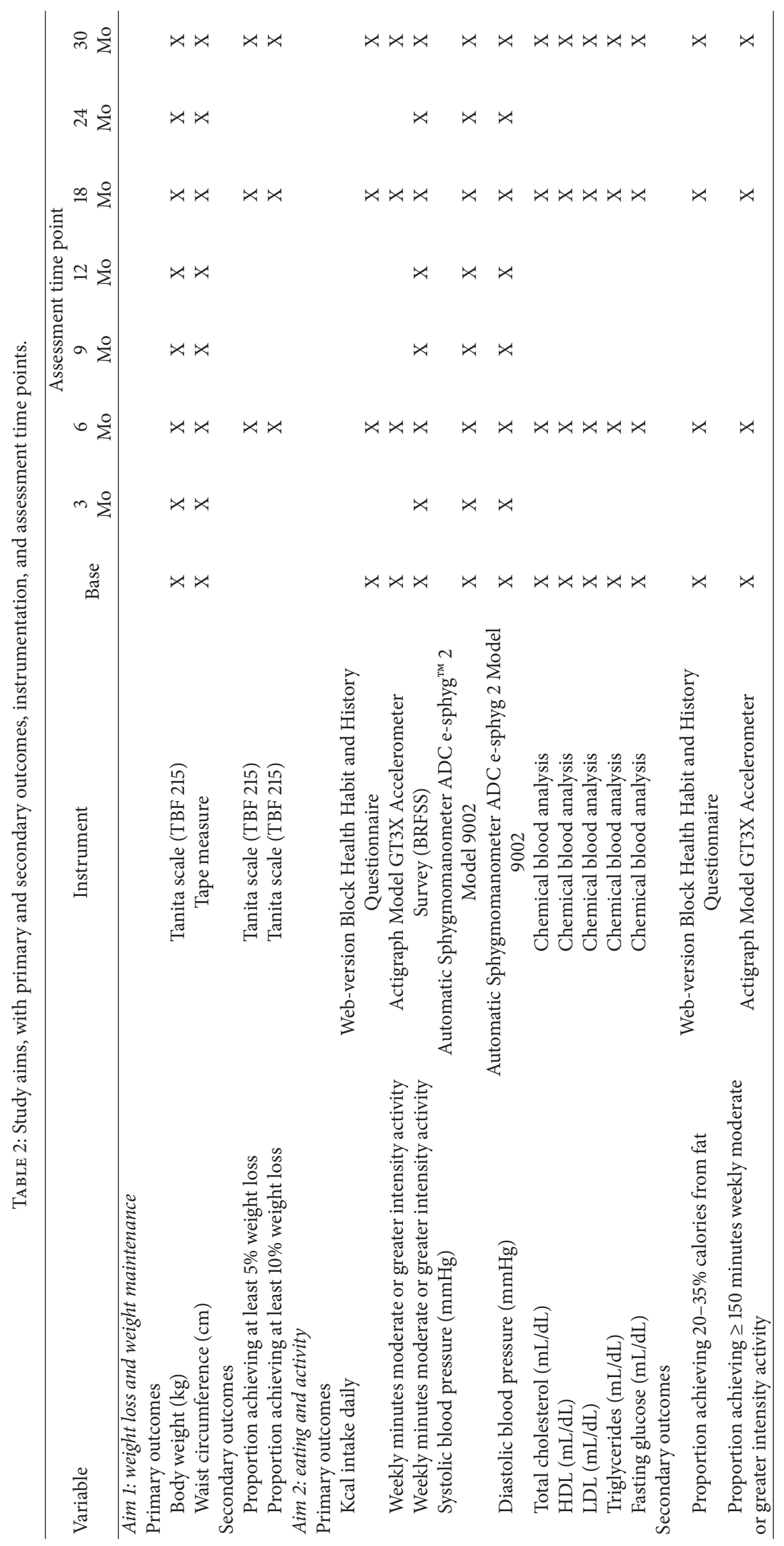


points of new primers posted by the peer-leader. For those in the WE group, monitoring of the number of emails sent by the professional email counselor and the replies from participants was logged; however, the planned protocol to use the email return receipt feature to monitor women's opening of email messages was found to be not feasible.

3.7. Sample Size. As detailed previously, the sample size of 306 was based on the most conservative planned test of group difference in average change in primary outcomes from beginning to end of a phase using generalized estimating equations [29]. This would provide power of at least .80 using $\alpha=.017$ for a two-tailed test of a mean difference of .45 standard deviations, the median effect size for weight and waist circumference found for this comparison in prior studies. Based on our pilot work, an effect of this magnitude was also plausible for group differences in change in physical activity outcomes, healthy eating behaviors, blood pressure, and lipids. The sample size also allowed for an estimated 25\% attrition over the course of the study. The revised analysis using linear mixed model methods to estimate a mixedeffects repeated-measures model maintained power of at least .80 with our observed data for the same effect size and planned comparisons.

In our National Institutes of Health study proposal, we followed an accepted strategy of estimating a priori power only for the analysis of primary outcomes, acknowledging that analyses of secondary outcomes were likely to have less power but would be conducted for their descriptive value. However, the power of a two-tailed $z$-test of pairwise differences in estimated proportions with $n=100$ per group and $\alpha=.017$ is .80 if the difference is approximately .22. The GEE analysis with imputed data, which also took into account dependence of observations across times, might be expected to have power of .80 for a somewhat smaller difference.

3.8. Data Analysis. The SPSS v.23 Missing Values Analysis module was used to evaluate missing data. Nearly all cases had complete data at baseline, with two women $(0.7 \%)$ missing actigraphy variables and $1(0.3 \%)$ missing lipids. Statistical comparisons of women with complete data $(n=$ 208 ) and those with at least one missing data point ( $n=$ 93) found the groups similar in baseline marital status, education, Internet access, comfort with using computers, waist circumference, and activity. However, women with missing data were approximately 2.4 years younger and $4.5 \mathrm{~kg}$ heavier and consumed 170 more calories per day at baseline. Weight loss for dropouts was not significantly different for completers as compared to noncompleters at 18 months $(.4 \mathrm{~kg}$ less, $p=0.879$ ).

Each intervention phase had distinct theoretically derived elements, and the primary analysis tested pairwise comparisons of the intervention groups on average change within each phase: (a) WO with WD using a one-sided test, (b) WO with WE using a one-sided test, and WD with WE using a two-sided test. Because these comparisons were nonorthogonal, each was tested at $\alpha=.017(.05 / 3)$.
A maximum likelihood approach was used for the analysis of the primary (continuous) outcomes. With fixed and common measurement times (time treated as categorical), a mixed-effects repeated-measures model can be fit using linear mixed model methods, specifying a random intercept and unstructured variance/covariance error matrix [54]. Partial cases are included in this analysis under the assumption that missing observations are missing at random (MAR). Each participant's data were analyzed according to her randomized assignment, regardless of adherence to protocol. Data from all cases and at all times (including intermediate assessments, if available) were used to estimate the model, which incorporated contrasts to test pairwise group differences from the beginning to the end of each phase. Supplemental tests of simple effects, conducted using a $p$ of 0.05 , were used to evaluate change within each group.

Secondary outcomes for aims 1 and 2 were attainment of criterion weight loss and eating and activity targets, dichotomous outcomes having nonnormal distributions. Thus, generalized estimating equations were used to perform the analysis. For the subset of outcomes needed in order to determine whether criteria were met, multiple imputation of missing data was carried out using SPSS Missing Values Analysis under the assumption that data were missing at random. Imputation was performed separately for each intervention group in order to preserve any interactions of group with the other variables. Two baseline variables were included as auxiliary variables: age, which had modest correlations with many variables, and perceived barriers to healthy eating, which was significantly associated with missingness. No variables were transformed prior to imputation [55]. A fully conditional specification (FCS) method was used for all variables, and 20 imputed datasets were created. After imputation, dichotomous variables indicating whether or not criteria were met were calculated. Using SAS 9.3, generalized estimating equations with a binomial error distribution and logit link were used to test pairwise group differences in the proportion of women meeting these goals at the end of each phase $(6,18$, and 30 months), accounting for the nonindependence across time. As with the linear mixed model, time was treated as categorical. PROC MIANALYZE in SAS was used to combine estimates across imputed datasets.

Intervention dosage was analyzed by phase with descriptive statistics, tabulating the frequency and percentage of women completers in each group by operationally defined dosage levels based on the number of weeks in which they logged on to the website. In addition, the correlation of number of logins and weight loss by 30 months was calculated. For the WD group, dosage of posting to the discussion board was also calculated.

\section{Results}

4.1. Participant Flow. Of 687 women assessed for trial eligibility, 323 women were enrolled and 301 women randomized after participating in the practice period and completing the baseline two visit (see Figure 1). Retention rates were $88.7 \%$, $76.5 \%$, and $71.8 \%$ between baseline and each of 6 months, 


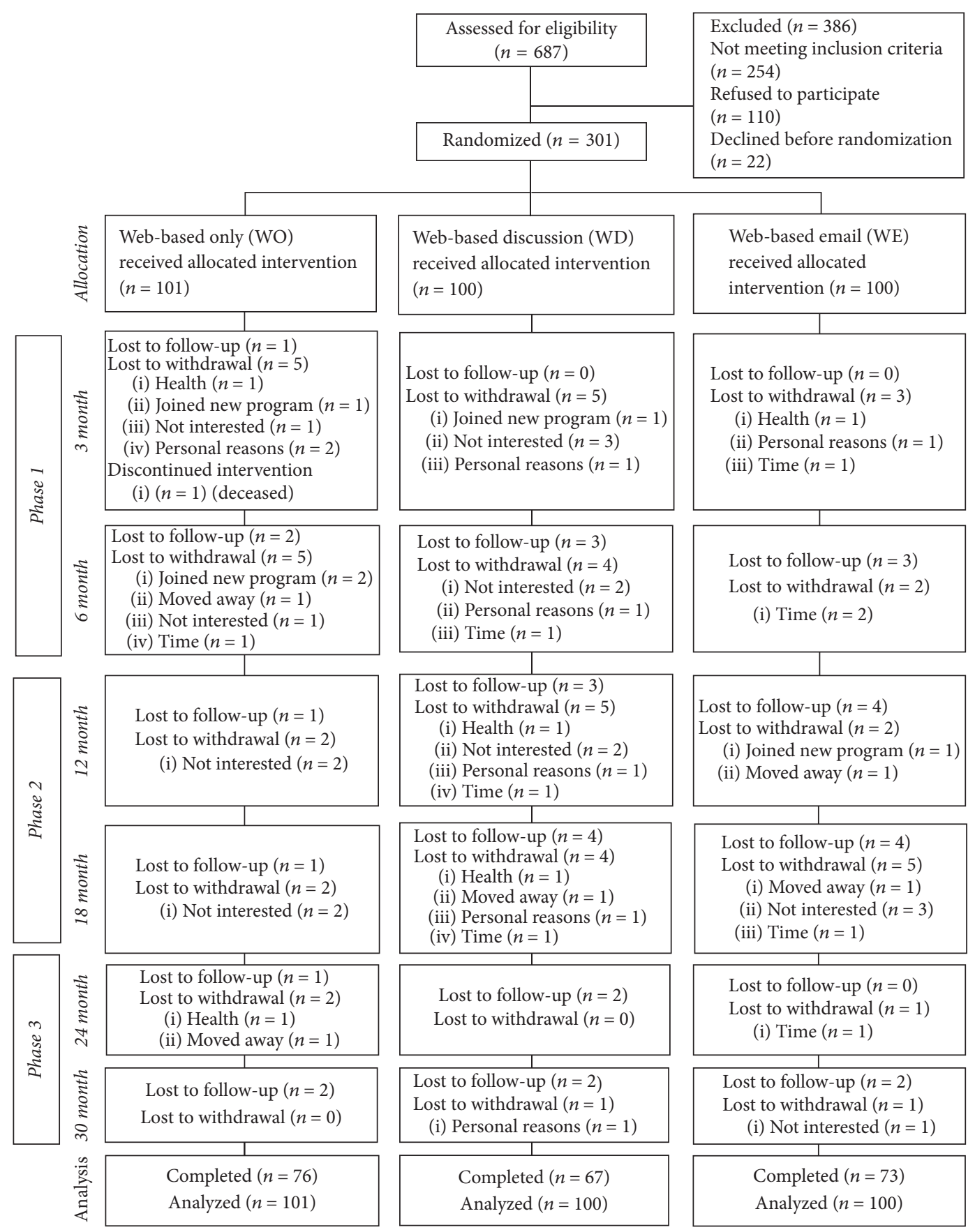

FIgURE 1: Participant flow diagram of the Women Weigh-in for Wellness clinical trial.

18 months, and 30 months, respectively, with no significant differences in retention rates across groups. Of those who dropped from the study, the primary reasons for withdrawal were lost to follow-up $(n=28)$, not interested/did not wish to continue $(n=11)$, and time commitment $(n=9)$.

4.2. Baseline Characteristics. The women were randomized into WO $(n=101)$, WD $(n=100)$, and WE $(n=100)$ groups. Baseline characteristics did not differ among the groups (see Table 3). Collectively, the mean age (SD) was 53.9
(6.9) years. Based upon BMI, 32 (10.6\%) were overweight, $143(47.5 \%)$ were obese I, 85 (28.2\%) were obese II, and 41 (13.6\%) were obese III. Baseline characteristics showed that the majority were Caucasian $(97.3 \% ; n=293)$, were employed full-time $(68.8 \% ; n=207)$, and had a college degree or higher education $(84.4 \% ; n=254)$. The majority $(77.1 \% ; n=232)$ reported having a household income $>\$ 40,000$. Overall, the women were healthy, with $106(35.2 \%)$ reporting having no comorbidities, $95(31.6 \%)$ reporting one comorbidity, and the remainder having two or more comorbidities. The most 
TABLE 3: Baseline characteristics of rural women by randomized group.

\begin{tabular}{|c|c|c|c|c|c|c|}
\hline \multirow[t]{2}{*}{ Variable } & \multicolumn{2}{|c|}{$\begin{array}{c}\text { Web-based } \\
\text { only (WO) } \\
n=101\end{array}$} & \multicolumn{2}{|c|}{$\begin{array}{c}\text { Web-based } \\
\text { discussion (WD) } \\
n=100\end{array}$} & \multicolumn{2}{|c|}{$\begin{array}{l}\text { Web-based } \\
\text { email (WE) } \\
n=100\end{array}$} \\
\hline & $n$ & $\%$ & $n$ & $\%$ & $n$ & $\%$ \\
\hline \multicolumn{7}{|l|}{ Race or ethnicity } \\
\hline White & 99 & $98 \%$ & 98 & $98 \%$ & 96 & $96 \%$ \\
\hline Hispanic & 1 & $1 \%$ & 1 & $1 \%$ & 1 & $1 \%$ \\
\hline Other & 1 & $1 \%$ & 1 & $1 \%$ & 2 & $1 \%$ \\
\hline No response & 0 & $0 \%$ & 0 & $0 \%$ & 1 & $1 \%$ \\
\hline \multicolumn{7}{|l|}{ Education } \\
\hline High school or lower & 20 & $20 \%$ & 12 & $12 \%$ & 15 & $15 \%$ \\
\hline Some college & 45 & $45 \%$ & 45 & $45 \%$ & 41 & $41 \%$ \\
\hline College grad or above & 36 & $35 \%$ & 43 & $43 \%$ & 44 & $44 \%$ \\
\hline \multicolumn{7}{|l|}{ Employment } \\
\hline Full time & 67 & $66 \%$ & 63 & $63 \%$ & 77 & $77 \%$ \\
\hline Part time & 18 & $18 \%$ & 26 & $26 \%$ & 9 & $9 \%$ \\
\hline \multicolumn{7}{|l|}{ Household income } \\
\hline$<\$ 20,000$ & 1 & $1 \%$ & 4 & $4 \%$ & 3 & $3 \%$ \\
\hline$\$ 20,000$ to $\$ 39,999$ & 15 & $15 \%$ & 15 & $15 \%$ & 13 & $13 \%$ \\
\hline$\$ 40,000$ to $\$ 59,999$ & 25 & $25 \%$ & 26 & $26 \%$ & 27 & $27 \%$ \\
\hline$\$ 60,000$ or higher & 52 & $52 \%$ & 49 & $49 \%$ & 51 & $51 \%$ \\
\hline \multicolumn{7}{|l|}{ Rural residency } \\
\hline Large rural & 56 & $55 \%$ & 63 & $63 \%$ & 70 & $70 \%$ \\
\hline Small rural & 10 & $10 \%$ & 10 & $10 \%$ & 4 & $4 \%$ \\
\hline Isolated & 33 & $33 \%$ & 27 & $27 \%$ & 26 & $26 \%$ \\
\hline \multicolumn{7}{|l|}{ Comorbid conditions } \\
\hline Diabetes & 3 & $3 \%$ & 5 & $5 \%$ & 6 & $6 \%$ \\
\hline Respiratory & 6 & $6 \%$ & 5 & $5 \%$ & 7 & $7 \%$ \\
\hline Arthritis & 28 & $28 \%$ & 21 & $21 \%$ & 31 & $31 \%$ \\
\hline $\begin{array}{l}\text { Other muscular } \\
\text { conditions }\end{array}$ & 7 & $7 \%$ & 10 & $10 \%$ & 15 & $15 \%$ \\
\hline Cancer & 11 & $11 \%$ & 9 & $9 \%$ & 7 & $7 \%$ \\
\hline Thyroid & 28 & $28 \%$ & 20 & $20 \%$ & 22 & $22 \%$ \\
\hline Cardiovascular & 3 & $3 \%$ & 1 & $1 \%$ & 4 & $4 \%$ \\
\hline General & 34 & $34 \%$ & 40 & $40 \%$ & 31 & $31 \%$ \\
\hline Smoking cigarettes & 1 & $1 \%$ & 4 & $4 \%$ & 7 & $7 \%$ \\
\hline \multicolumn{7}{|l|}{ General health } \\
\hline Excellent to very good & 38 & $38 \%$ & 26 & $26 \%$ & 40 & $40 \%$ \\
\hline Good & 54 & $54 \%$ & 60 & $60 \%$ & 47 & $47 \%$ \\
\hline Poor to fair & 9 & $9 \%$ & 13 & $13 \%$ & 13 & $13 \%$ \\
\hline \multicolumn{7}{|l|}{ BMI category $\left(\mathrm{kg} / \mathrm{m}^{2}\right)$} \\
\hline Overweight (25-29.9) & 12 & $12 \%$ & 7 & $7 \%$ & 13 & $13 \%$ \\
\hline Obese I (30-34.9) & 50 & $50 \%$ & 49 & $49 \%$ & 44 & $44 \%$ \\
\hline Obese II (35-39.9) & 24 & $24 \%$ & 30 & $30 \%$ & 31 & $31 \%$ \\
\hline Obese III ( $\geq 40$ to 45 ) & 15 & $15 \%$ & 14 & $14 \%$ & 12 & $12 \%$ \\
\hline
\end{tabular}


common comorbidities were the general health issues of anxiety, depression, or migraines $(34 \%, n=105)$ and arthritis $(26.6 \%, n=80)$.

\subsection{Aim 1: Weight}

4.3.1. Primary Outcomes. The intervention groups did not differ significantly from each other in mean change on either of the primary outcomes (body weight and waist circumference). This finding was consistent across all phases and for all pairwise comparisons (see Tables 4 and 5). Considering simple effects within group, all means on both primary outcomes decreased significantly from baseline to 6 months. Across the groups, estimated mean weight loss at 6 months ranged from 4.0 to $5.8 \mathrm{~kg}$, representing from $4.2 \%$ to $6.2 \%$ of initial body weight. Mean decreases in waist circumference ranged from 4.6 to $6.2 \mathrm{~cm}$. However, from 6 months to 18 months, there was significant increase in all groups, with average weight increasing by approximately $2 \mathrm{~kg}$ or from onethird to nearly one-half of phase 1 loss. On average, there was further significant weight gain of about $1 \mathrm{~kg}$ from 18 months to 30 months. Waist circumference also significantly increased during phase 2 .

4.3.2. Secondary Outcomes. The estimated percentage of women in each group who lost at least $5 \%$ of their baseline weight by the end of phase 1 was $42 \%$ in the WO group, $38 \%$ in the WD group, and $51 \%$ in the WE group. Percentages for meeting the criterion of at least $10 \%$ weight loss were $24 \%$, $19 \%$, and $29 \%$, respectively. None of the pairwise comparisons were significant for these outcomes in any of the three phases. The proportion meeting each of these criteria dropped by about one-third from the end of phase 1 to the end of phase 2 , but almost no further change occurred during phase 3 (see Table 6).

\subsection{Aim 2: Eating and Activity}

4.4.1. Primary Outcomes. Within each phase, there were no significant pairwise comparisons for any of the primary outcomes (Kcal intake, weekly minutes of moderate or greater intensity activity, blood pressure, or lipids), with the exception of a larger improvement in HDL seen in the WO group compared to the WE group in phase 1. Effects favoring the WO group had not been hypothesized (see Table 5).

Simple main effects tests for eating and activity measures showed the desired change that occurred in self-reported activity, kcal intake, triglycerides, and HDL during phase 1. Systolic and diastolic blood pressure showed significant decreases in phase 1 in the WE group, but only systolic blood pressure decreased in the WO group. Both groups maintained these improvements in phases 2 and 3.

4.4.2. Secondary Outcomes. A significantly higher proportion of the WE group than the WD group met the standards for $\%$ of calories from fat at 6 months (estimated proportions of .56 versus .38). No differences were observed between groups in the proportion of individuals meeting the physical activity targets, whether measured by Actigraph or selfreport, in any of the phases.

4.5. Website Usage and Weight Change. Logins by week of new web-intervention content were used to estimate the dosage of web intervention received (Table 7). The pattern of participation in the basic website was similar among women in all groups for phase 1 , with $69.0 \%, 65.9 \%$, and $69.6 \%$ of $6-$ month completers in WO, WD, and WE groups, respectively, logging in for $\geq 50 \%$ dosage. The dosage of web intervention received declined by approximately half during phase 2 and two-thirds in phase 3 . Weight change was significantly related to the website utilization $(r=-0.25, p<0.01)$, with greater participation correlated with greater success with weight loss and weight maintenance over the trial.

Among women in the WD group, nearly half (45\%) viewed and/or posted on the discussion board for a dosage of $50 \%$ or more when new discussion board primers were made available during phase 1 . Women's use of the discussion board dropped dramatically during phase 2 , with only about $22 \%$ having a dosage of $50 \%$ or more. During phase 3 , the majority of women $(58.2 \%)$ in the WD group never viewed the discussion board.

For the WE group, the intervention delivery was 99.9\%, meaning that the targeted number of messages was sent to the participants at the scheduled time. Eighty-nine percent and $69 \%$ of WE participants sent email responses to the email counselor during phases 1 and 2, respectively. During phase 3, the WE group no longer received emails from the counselor, but $16 \%$ of these women sent emails to the counselor during that 1-year self-management phase.

\section{Discussion}

The Women Weigh-in for Wellness community-based clinical trial compared the effectiveness of a web-based only intervention (WO) with two web-based interventions with additional elements (WD and WE) on achieving initial weight loss (baseline to 6 months), guided weight loss and weight maintenance ( 6 to 18 months), and self-management of weight maintenance (18 to 30 months), with findings that, on average, women from rural communities in all groups achieved the majority of weight loss at 6 months (representing $4.2 \%$ to $6.2 \%$ of initial weight loss), with gradual regain of approximately half the weight lost by 30 months. There were no differences in this outcome among the three web-based groups (WO, WD, and WE) within any of the phases. The 6month estimated group mean weight losses ranged between 4.0 and 5.8 kilograms, with 42\% (WO), 38\% (WD), and $51 \%$ (WE) of women meeting the 6 -month target of $\geq 5 \%$ of initial body weight loss. All three groups showed a drop of approximately 10 percentage points in the number of women meeting the $\geq 5 \%$ loss criterion by 30 months.

The observed pattern of our women achieving the most rapid weight loss during phase 1 guided weight loss (baseline to 6 months), with women showing a slow rate of regain during phase 2 (6 to 18 months) and phase 3 (18 to 30 months), 


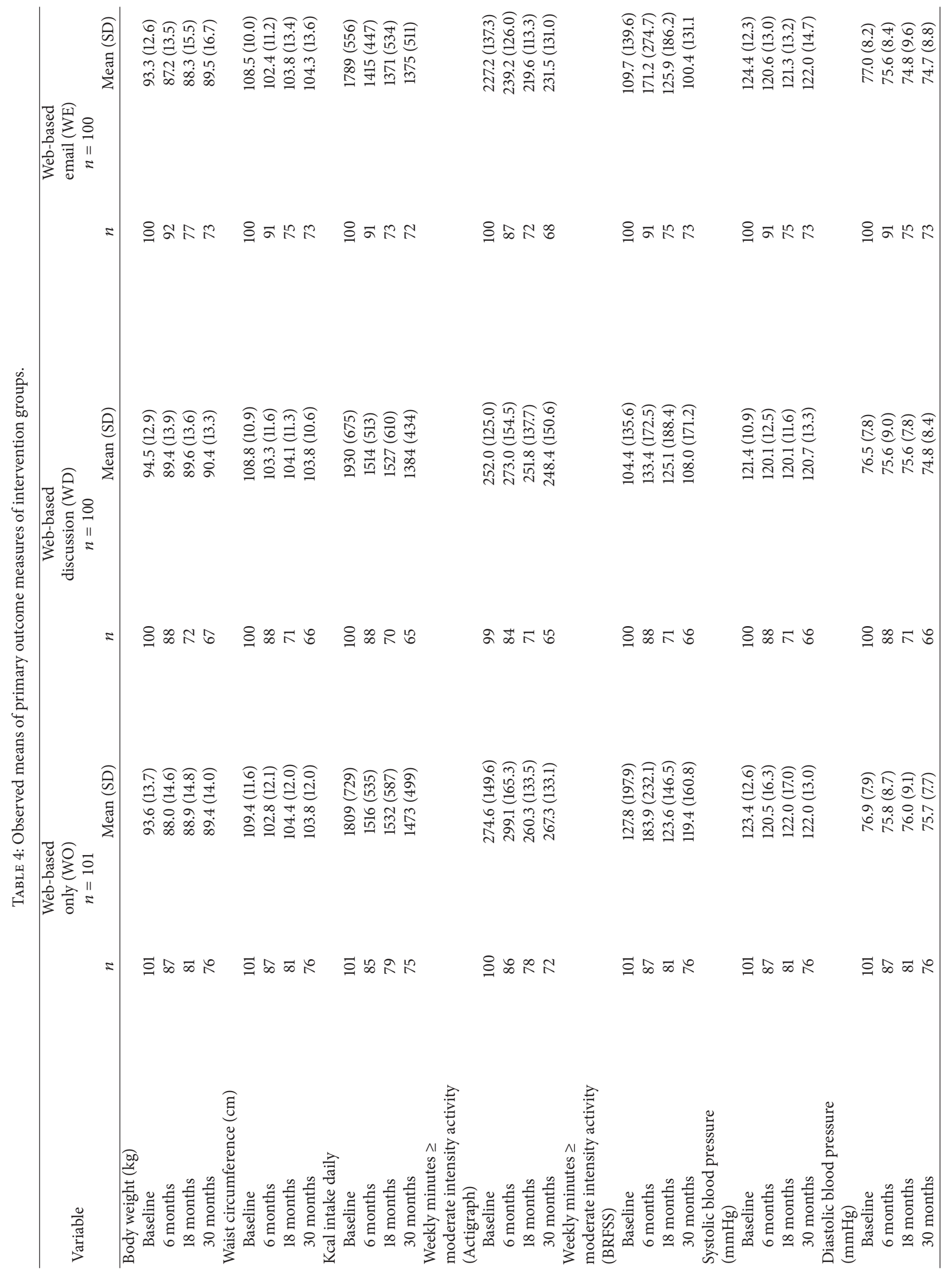




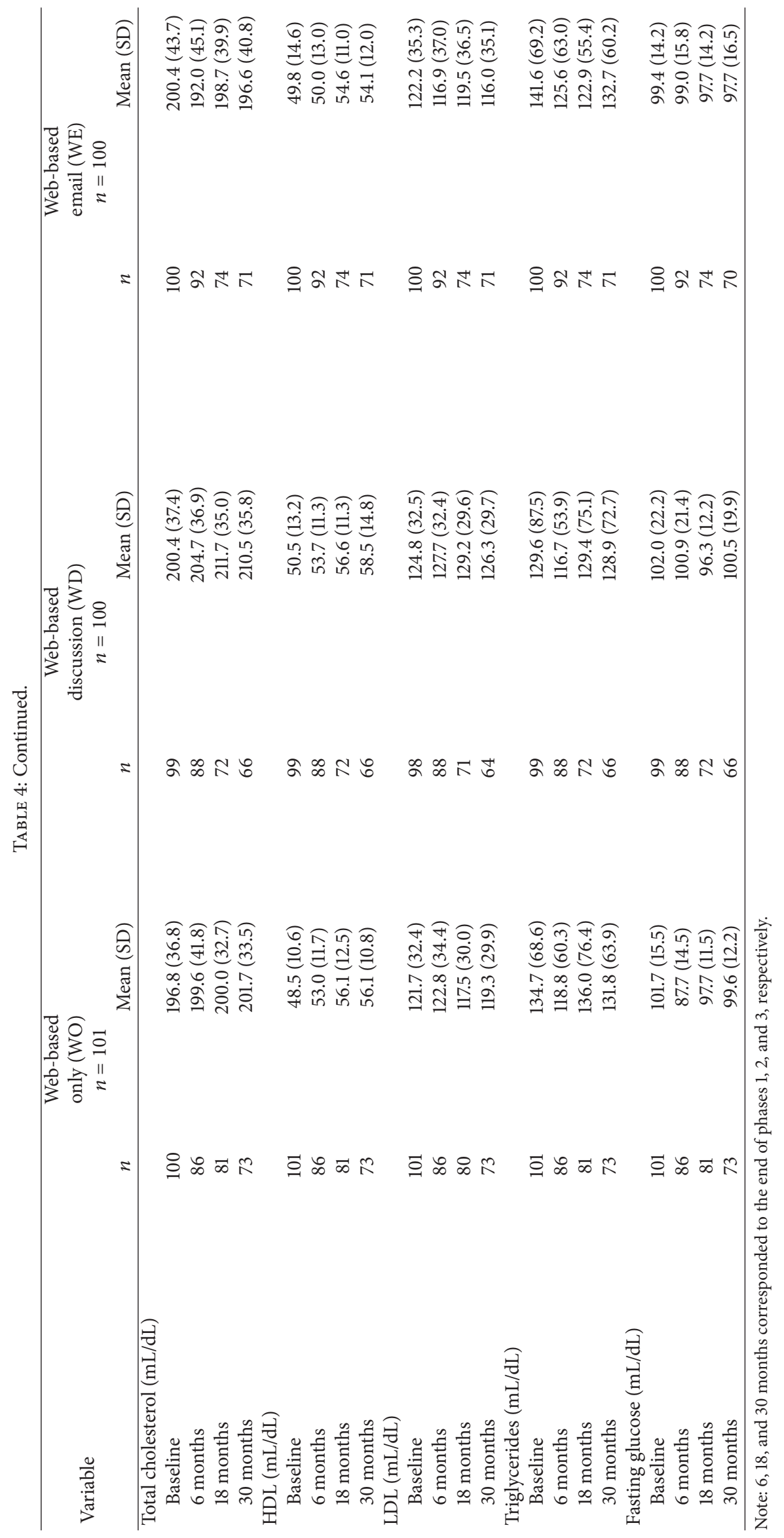




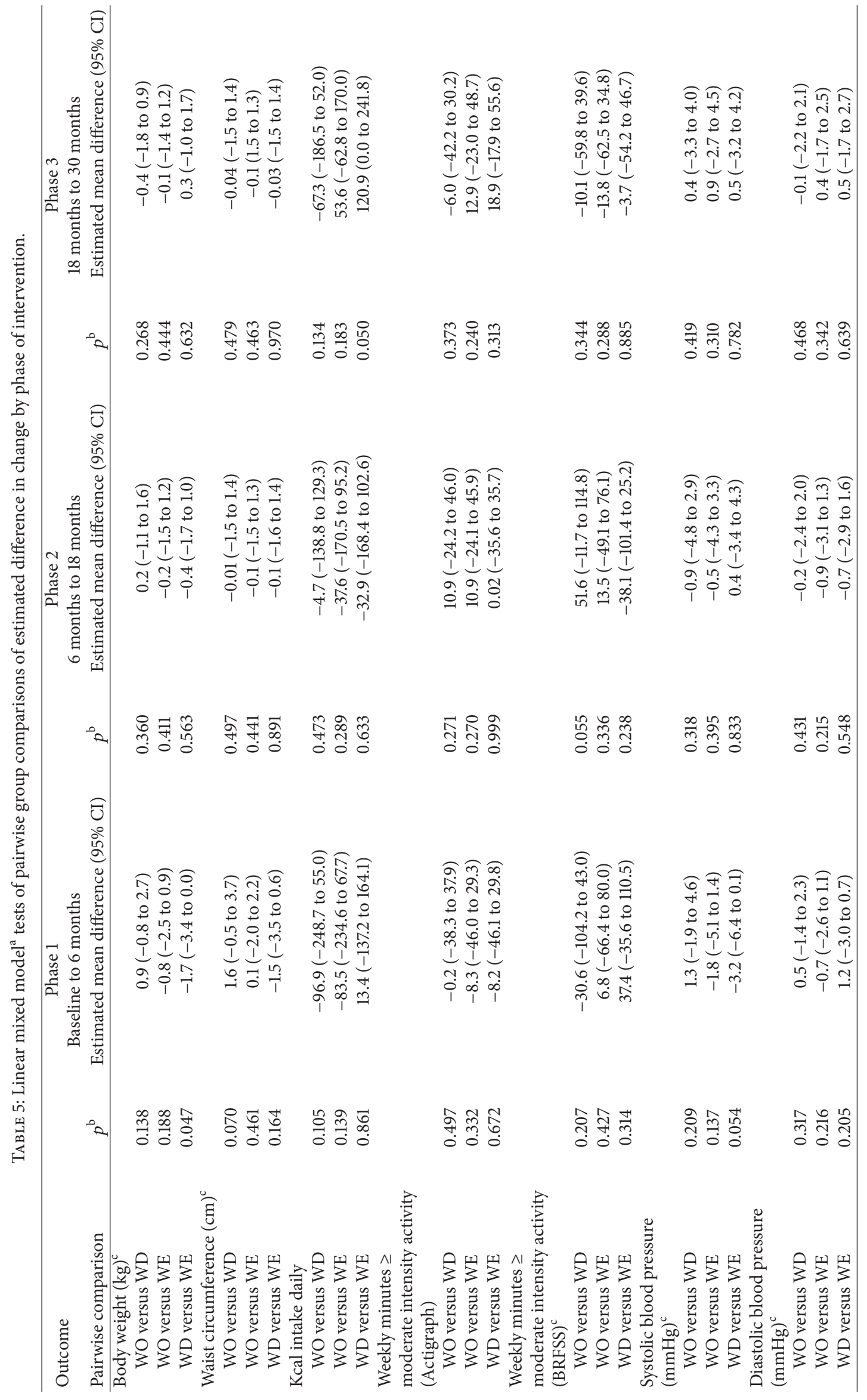




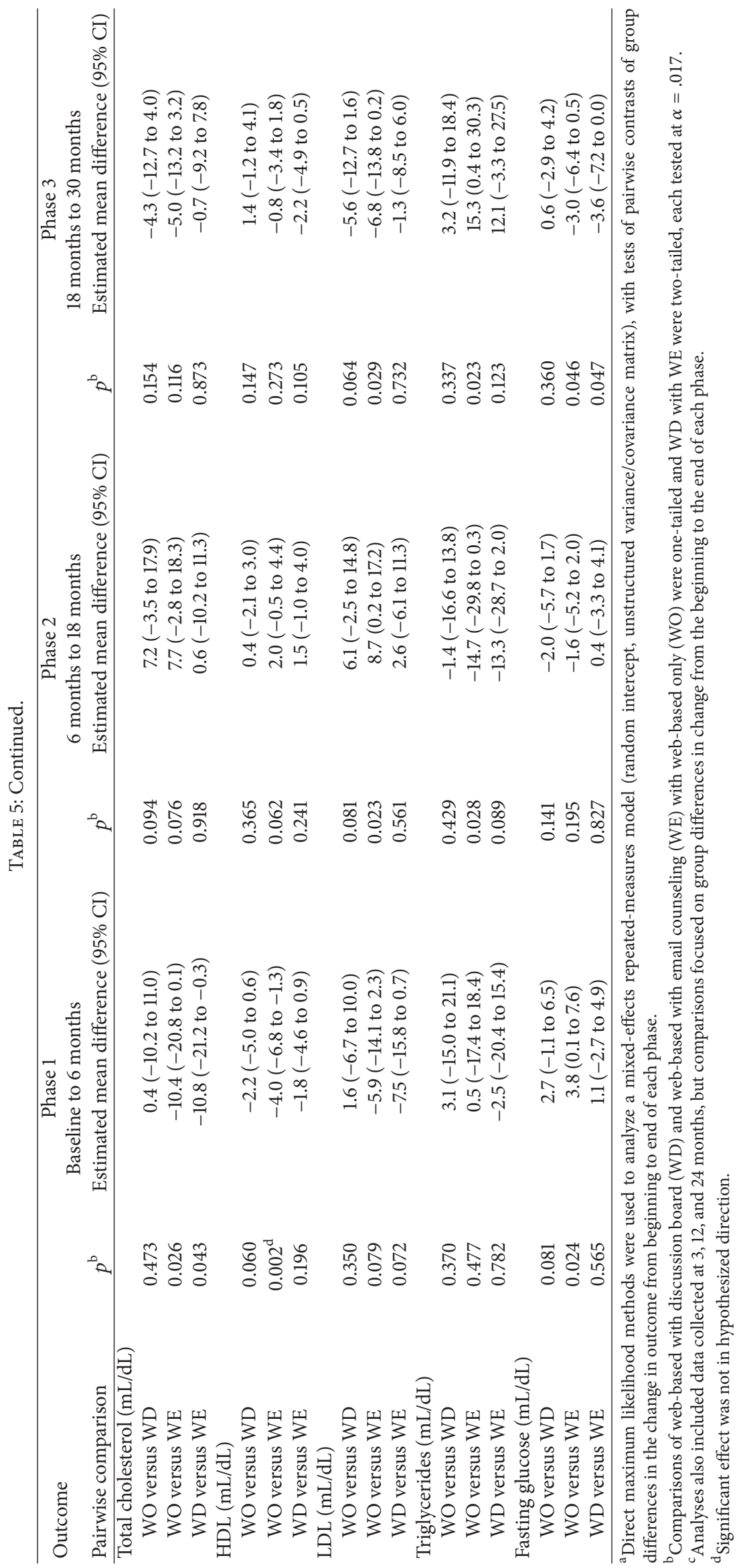




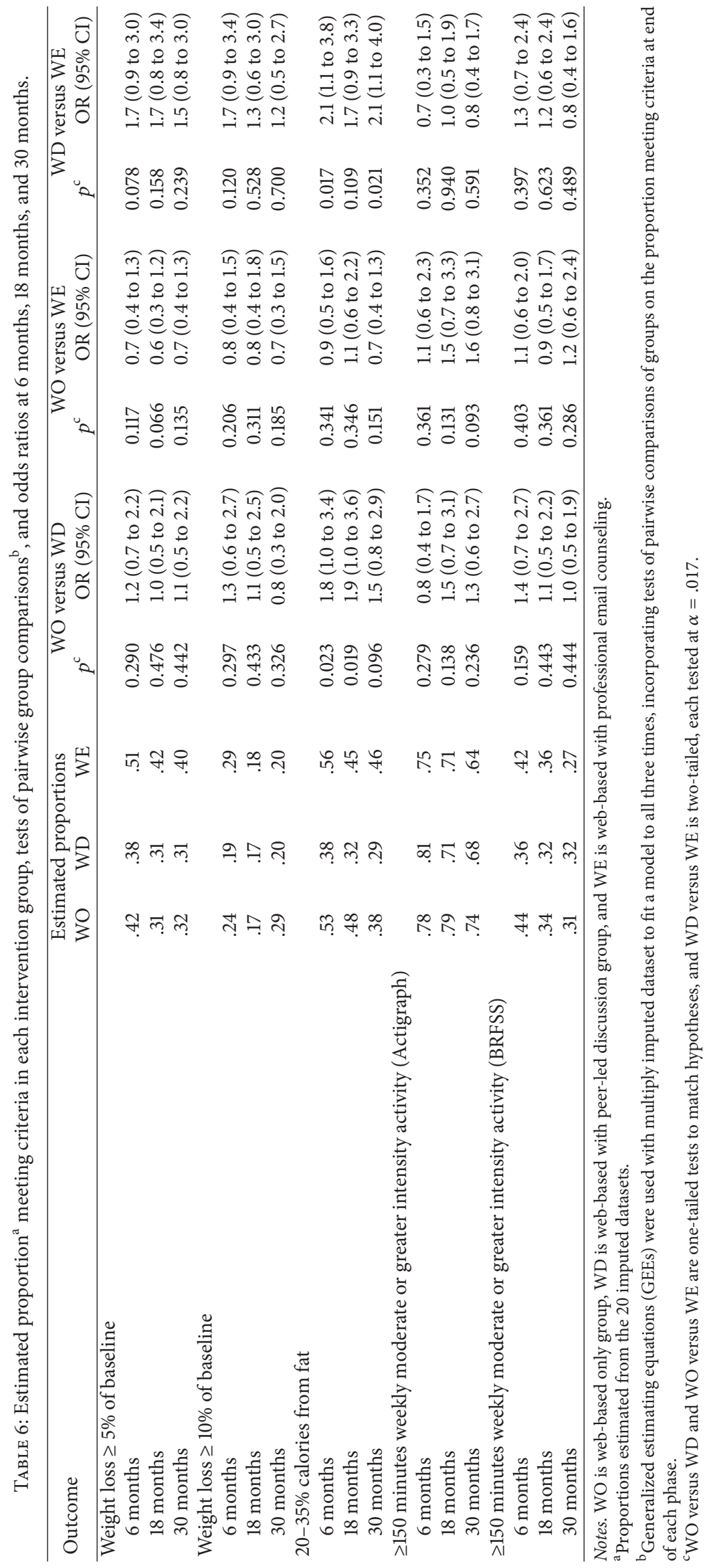




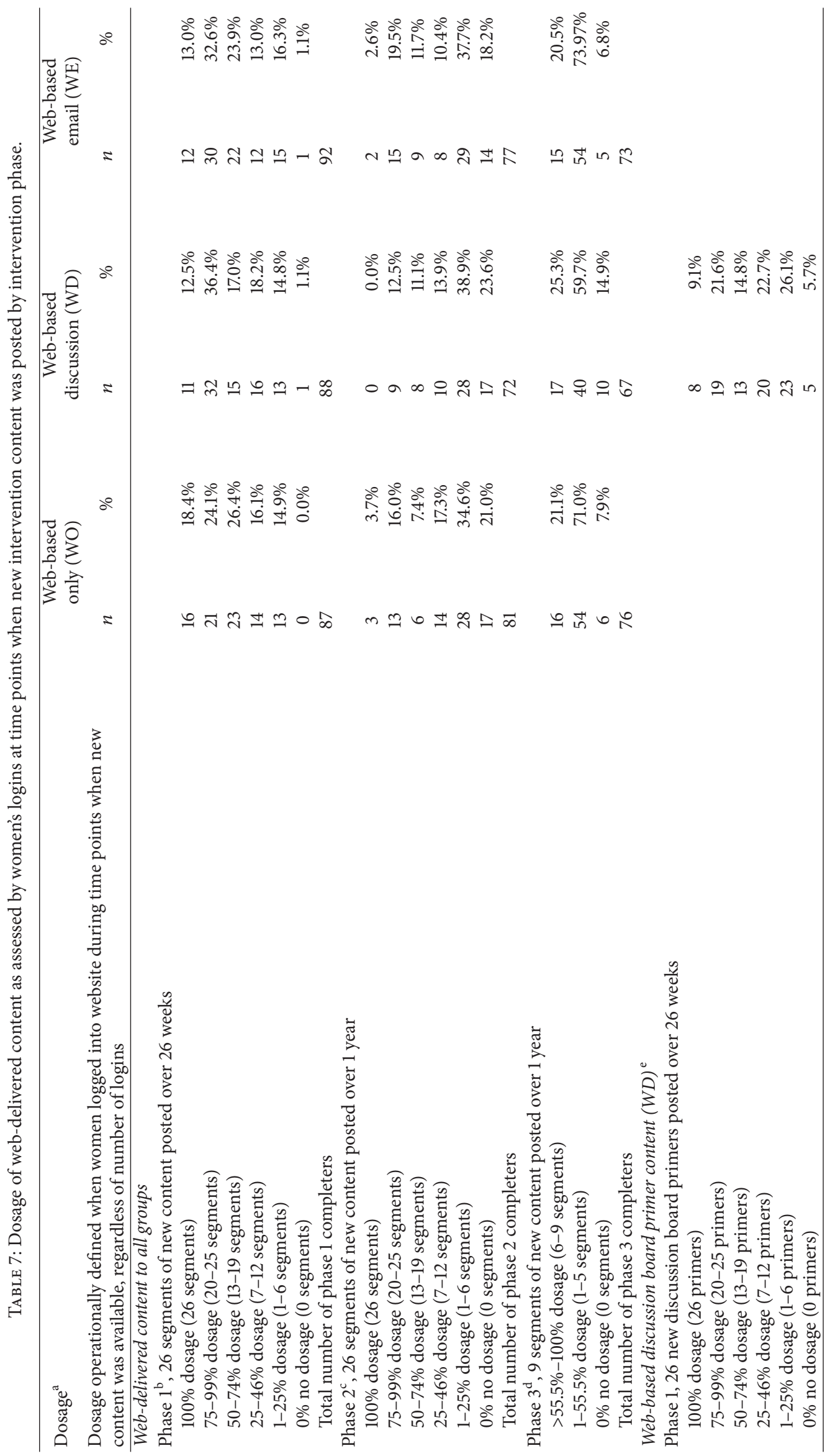




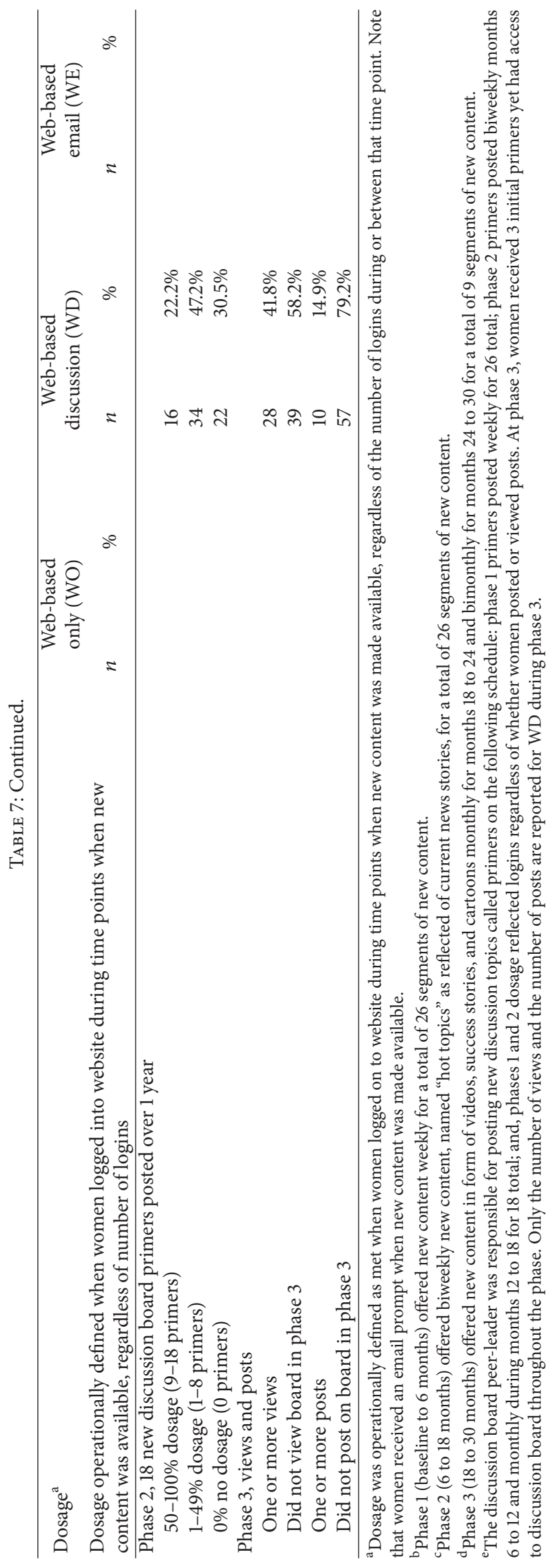


is consistent with findings of a systematic review and metaanalysis of weight loss clinical trials with a minimum oneyear follow-up conducted by Franz et al. [56]. As noted in a Cochrane Systematic Review of web-based weight loss and weight maintenance interventions [7], the different rate of weight change suggests that weight loss and weight loss maintenance interventions need to be considered separately, as was done in this study.

The amount of weight change among women in this study, particularly at the 6-month endpoint, is consistent with the ranges reported among other purely web-based studies $[17,20,26]$. Overall, an estimated $43.7 \%, 34.7 \%$, and $34.3 \%$ of women at 6,18 , and 30 months, respectively, met the weight loss target of $\geq 5 \%$, the amount recommended for health benefits. Although weight loss was modest, there is evidence that suggests that some degree of weight loss, even if it is not sustained beyond 6 months, may prove beneficial for blood pressure [11]. Weight losses below 5\% may be of clinical significance on a public level, as for each $\mathrm{kg}$ of weight loss, a meta-analysis of 25 studies shows observed blood pressure reductions of $1.1 \mathrm{~mm} \mathrm{Hg}$ and $0.9 \mathrm{~mm} \mathrm{Hg}$ for systolic and diastolic blood pressures, respectively [57].

WO did not differ from WD and WE in mean change in body weight or waist circumference across all phases, a finding that was unexpected and counter to our hypotheses. These results might be due to the robust nature of the webbased intervention used by all three groups. The web-based intervention was comprehensive in that it included a structured weight loss and weight maintenance plan, delivered updated content frequently, provided email prompts when new content was updated, and included self-monitoring for eating, activity, and goal setting across all phases. Such comprehensive web-based features promote greater weight reduction than those with basic informational elements [9, 58]. The frequency of the assessments, found by others to enhance achievement of weight loss or weight maintenance, might have had an unintended effect as intervention boosters particularly for the WO group [16].

The combination of frequency of our assessments with the email prompts when new web content appeared may have been a factor in our relatively high retention rate for all three groups over the 30 months, being $88 \%$ or greater at 6 months, with declines to $69 \%$ or greater by 30 months. Compared to other studies, our retention was relatively high given the long intervention period. The literature cites a wide range of retention rates for purely web-delivered weight loss, from $6 \%$ to $35 \%[58,59]$ over 12 weeks, with others having higher retention of $80 \%$ after 12 months of intervention [16].

Issues with long-term sustainability and nonusage attrition are common in web-based interventions and are problems that appear to increase over time [60]. Reasons for disengagement may be motivational, failure to achieve weight loss, or lack of interest in the features or content of the website, which may lead to boredom [60]. Consistent with the literature [61], success at weight loss and weight loss maintenance among our women was correlated with the level of women's engagement by our proxy of intervention dosage, with the pattern of website dosage received being similar across all groups, declining over the three phases.
Our method of tracking dosage, being percent of women who logged in at least once when new content was delivered (i.e., weekly, biweekly, or monthly), was similar to that of Cussler and colleagues [15], who defined dosage as the percentage of participants who used the feature at least once per week. Several groups of researchers [7, 59] noted the challenges in comparing adherence data, as there is no standardization for adherence overall, though studies report a positive association between participant adherence and weight change.

Women in the WD group had relatively low engagement in the discussion board feature initially during phase 1 with continued declines over phases 2 and 3. The reasons for this low engagement in the discussion board are unclear as our prior 3-month pilot study comparing WO to WD showed enhanced engagement and weight loss in the WD group. As the women's enrollment in this large trial occurred over a one-year period, it was possible that the women never sensed they were part of a cohort, or they first entered the discussion board at times when the content was not helpful to their given needs or interests, or the technology frustrated them. As noted in a systematic review of online peer-to-peer interactions, there is a lack of robust evidence on peer-to-peer online support, though this does not mean that virtual social communities have no effect, as little is known about factors that might influence outcomes, such as moderator influences and/or individual and group interests [22].

In contrast, women in the WE group appeared more engaged. The percentage of women voluntarily responding to counselor emails was highest during phase $1(89 \%)$ and declined slightly during phase $2(69 \%)$. In phase $3,16 \%$ of women self-initiated an email to counselor when no routine counselor emails were sent. A potential reason for this level of engagement may be attributed to the personalization that is perceived with individualized emails, a finding observed by others $[7,58]$.

The strengths of this study included its design, comparing web-based only with two other web-based interventions with enhanced features among a population of underserved and understudied women from rural communities. The study included successful recruitment and retention of a large cohort of women from rural communities over 30 months, with nearly half from small rural or isolated rural areas. Analyses of primary outcomes were adequately powered (.80) for a moderate effect size consistent with prior weight loss and maintenance comparisons of interventions. For secondary outcomes, power had not been estimated a priori, but the size of the group difference in proportion needed to be found significant appears to also be meaningful. The uniqueness of this intervention is its inclusion of three phases, providing interventions that targeted guided weight loss, followed by longer intervention periods of 12 months each for guided weight maintenance and self-managed weight maintenance, and tracking women's engagement. The study design and implementation included features established for reporting eHealth interventions [62], which were published after this trial started enrolling participants. 
Limitations included the majority of the women being from a relatively high socioeconomic background, commonly reported in other web-based interventions, which may limit generalizability since the findings may not be representative of the population of overweight and obese women from rural communities. Another important limitation is that we cannot know to what extent missing observations were truly missing at random. The nonsignificant difference for weight loss between completers and noncompleters at 18 months provides some assurance that the MAR assumption may be tenable.

Contamination may have occurred between groups due to the women's familiarity and sharing with other women in the rural areas of residence. Though the nurses were blinded to the women's group assignment and to the intervention, the frequent contacts could have influenced the women's behavior for achieving weight loss or influenced retention. The issue of decreased or passive engagement with the intervention over time is common in web-based interventions. Additional analysis of women's engagement with specific website features associated with successful weight loss and weight maintenance may prove insightful.

The implications of this research are that women from rural communities were willing and able to participate in long-term web-based interventions for weight loss and weight maintenance. Future studies might focus on recruiting women more representative of the population, refining the interventions to encourage weight maintenance, and focusing on the cost-effectiveness of implementing webbased only interventions compared to those with supplemental features.

\section{Conclusions}

This study supports that women from rural communities with overweight or obesity were willing to participate in webbased interventions, either web-based only or web-based with supplemental elements, with an estimated $42 \%$ (WO), $38 \%$ (WD), and 51\% (WE) being able to achieve clinically relevant weight loss of $\geq 5 \%$ by six months, with weight regain by half at 30 months, though the WD and WE groups did not result significantly in more improvement compared to the WO group, potentially due to the robust nature of the web-based intervention. Though weight change was modest, the use of web-based interventions may be clinically relevant for reaching rural women on a public health level, as small reductions in weight have been shown to have health benefits. As women from rural communities may have few resources for weight management, web-based weight loss and weight maintenance programs may be potentially important as an alternative venue.

\section{Conflicts of Interest}

Drs. Hageman and Pullen declare a patent copyright on the "Banding Together for Strength" DVD used in the intervention component of the clinical trial from which participant baseline data were analyzed for this manuscript.

\section{Acknowledgments}

This study was funded by a National Institute of Nursing Research, National Institutes of Health Grant (Grant no. R01 NR010589), with supplemental funding from a Research Support Fund Grant from The Nebraska Medical Center and the University of Nebraska Medical Center for lipid and glucose testing and from The Hygenic Corporation Thera-Band Academy for equipment support. Appreciation is extended to Matt Gotschall, President, Central Community CollegeColumbus, NE, for agreeing to provide physical space for the project and to Deepak Khazanchi, Associate Dean, College of Information Science and Technology, University of Nebraska-Omaha, Omaha, NE, for agreeing to design the intervention website and the database. The authors thank Maureen Oberdorfer and Michelle L. Potter, who served as project coordinators, and Paul J. Dizona for informational technology and data analyst support. Their gratitude and appreciation is extended to Kristin Gregerson and Laura Dewitt for their roles as peer-leader for the discussion board intervention group and professional email counseling group, respectively. The authors thank Carolyn Korth, Heather Speicher, and Jessica M. Hanson, research nurses, who conducted participant assessments at Central Community College in Columbus. Appreciation is extended to Katie Korth for her role in participant recruitment and Amy Onstott for her assistance in screening potential participants. The authors would like to thank Veronica Myers, Dreyson Solano, and Zac Fowler from UNO for their programming for the website and developing the website database.

\section{References}

[1] C. A. Befort, N. Nazir, and M. G. Perri, "Prevalence of obesity among adults from rural and urban areas of the United States: findings from NHANES (2005-2008)," Journal of Rural Health, vol. 28, no. 4, pp. 392-397, 2012.

[2] S. A. Feresu, W. Zhang, S. E. Puumala, F. Ullrich, and J. R. Anderson, "The frequency and distribution of cardiovascular disease risk factors among Nebraska women enrolled in the WISEWOMAN screening program," Journal of Women's Health, vol. 17, no. 4, pp. 607-617, 2008.

[3] T. Trivedi, J. Liu, J. Probst, A. Merchant, S. Jones, and A. B. Martin, "Obesity and obesity-related behaviors among rural and urban adults in the USA," Rural and Remote Health, vol. 15 , no. 4 , article $3267,2015$.

[4] C. H. Pullen, S. N. Walker, P. A. Hageman, L. S. Boeckner, and M. K. Oberdorfer, "Differences in eating and activity markers among normal weight, overweight, and obese rural women," Women's Health Issues, vol. 15, no. 5, pp. 209-215, 2005.

[5] S. C. Folta, A. H. Lichtenstein, R. A. Seguin, J. P. Goldberg, J. F. Kuder, and M. E. Nelson, "The strongwomen-healthy hearts program: reducing cardiovascular disease risk factors in rural sedentary, overweight, and obese midlife and older women," American Journal of Public Health, vol. 99, no. 7, pp. 1271-1277, 2009.

[6] National Institutes of Health, "Strategic plan for NIH obesity research: a report of the NIH obesity task force," Tech. Rep., The U.S. Department of Health and Human Services, Washington, DC, USA, 2011. 
[7] L. S. Wieland, L. Falzon, C. N. Sciamanna et al., "Interactive computer-based interventions for weight loss or weight maintenance in overweight or obese people," Cochrane Database of Systematic Reviews, vol. 8, Article ID CD007675, 2012.

[8] E. Brindal, J. Freyne, I. Saunders, S. Berkovsky, G. Smith, and M. Noakes, "Features predicting weight loss in overweight or obese participants in a web-based intervention: randomized trial," Journal of Medical Internet Research, vol. 14, no. 6, article e173, 2012.

[9] L. C. H. Raaijmakers, S. Pouwels, K. A. Berghuis, and S. W. Nienhuijs, "Technology-based interventions in the treatment of overweight and obesity: a systematic review," Appetite, vol. 95, pp. 138-151, 2015.

[10] F. X. Pi-Sunyer, "How effective are lifestyle changes in the prevention of type 2 diabetes mellitus?" Nutrition Reviews, vol. 65, no. 3, pp. 101-110, 2007.

[11] V. J. Stevens, E. Obarzanek, N. R. Cook et al., "Long-term weight loss and changes in blood pressure: results of the trials of hypertension prevention, phase II," Annals of Internal Medicine, vol. 134, no. 1, pp. 1-11, 2001.

[12] P. S. MacLean, R. R. Wing, T. Davidson et al., "NIH working group report: innovative research to improve maintenance of weight loss," Obesity, vol. 23, no. 1, pp. 7-15, 2015.

[13] L. P. Svetkey, V. J. Stevens, P. J. Brantley et al., "Comparison of strategies for sustaining weight loss: the weight loss maintenance randomized controlled trial," The Journal of the American Medical Association, vol. 299, no. 10, pp. 1139-1148, 2008.

[14] A. Khaylis, T. Yiaslas, J. Bergstrom, and C. Gore-Felton, "A review of efficacious technology-based weight-loss interventions: five key components," Telemedicine Journal and E-Health, vol. 16, no. 9, pp. 931-938, 2010.

[15] E. C. Cussler, P. J. Teixeira, S. B. Going et al., "Maintenance of weight loss in overweight middle-aged women through the internet," Obesity, vol. 16, no. 5, pp. 1052-1060, 2008.

[16] V. J. Stevens, K. L. Funk, P. J. Brantley et al., "Design and implementation of an interactive website to support longterm maintenance of weight loss," Journal of Medical Internet Research, vol. 10, no. 1, article el, 2008.

[17] D. F. Tate, R. R. Wing, and R. A. Winett, "Using internet technology to deliver a behavioral weight loss program," The Journal of the American Medical Association, vol. 285, no. 9, pp. 1172-1177, 2001.

[18] D. F. Tate, E. H. Jackvony, and R. R. Wing, "Effects of internet behavioral counseling on weight loss in adults at risk for type 2 diabetes: a randomized trial," The Journal of the American Medical Association, vol. 289, no. 14, pp. 1833-1836, 2003.

[19] D. F. Tate, E. H. Jackvony, and R. R. Wing, "A randomized trial comparing human e-mail counseling, computer-automated tailored counseling, and no counseling in an internet weight loss program," Archives of Internal Medicine, vol. 166, no. 15, pp. 1620-1625, 2006.

[20] K. H. Webber, D. F. Tate, and J. Michael Bowling, "A randomized comparison of two motivationally enhanced Internet behavioral weight loss programs," Behaviour Research and Therapy, vol. 46, no. 9, pp. 1090-1095, 2008.

[21] M. G. Perri, M. C. Limacher, P. E. Durning et al., "Extended-care programs for weight management in rural communities: The Treatment of Obesity in Underserved Rural Settings (TOURS) randomized trial," Archives of Internal Medicine, vol. 168, no. 21, pp. 2347-2354, 2008.

[22] G. Eysenbach, J. Powell, M. Englesakis, C. Rizo, and A. Stern, "Health related virtual communities and electronic support groups: systematic review of the effects of online peer to peer interactions," British Medical Journal, vol. 328, no. 7449, pp. 1166-1169, 2004.

[23] J. M. Gabriele, B. D. Carpenter, D. F. Tate, and E. B. Fisher, "Directive and nondirective e-coach support for weight loss in overweight adults," Annals of Behavioral Medicine, vol. 41, no. 2, pp. 252-263, 2011.

[24] J. Harvey-Berino, S. J. Pintauro, and E. C. Gold, “The feasibility of using internet support for the maintenance of weight loss," Behavior Modification, vol. 26, no. 1, pp. 103-116, 2002.

[25] J. Harvey-Berino, S. Pintauro, P. Buzzell, and E. C. Gold, "Effect of internet support on the long-term maintenance of weight loss," Obesity Research, vol. 12, no. 2, pp. 320-329, 2004.

[26] B. C. Gold, S. Burke, S. Pintauro, P. Buzzell, and J. HarveyBerino, "Weight loss on the web: a pilot study comparing a structured behavioral intervention to a commercial program," Obesity, vol. 15, no. 1, pp. 155-164, 2007.

[27] C. H. Pullen, P. A. Hageman, L. Boeckner, S. N. Walker, and M. K. Oberdorfer, "Feasibility of internet-delivered weight loss interventions among rural women ages 50-69," Journal of Geriatric Physical Therapy, vol. 31, no. 3, pp. 105-112, 2008.

[28] C. L. Melvin, G. Corbie-Smith, S. K. Kumanyika et al., "Developing a research agenda for cardiovascular disease prevention in high-risk rural communities," American Journal of Public Health, vol. 103, no. 6, pp. 1011-1021, 2013.

[29] P. A. Hageman, C. H. Pullen, M. Hertzog, L. S. Boeckner, and S. N. Walker, "Web-based interventions for weight loss and weight maintenance among rural midlife and older women: protocol for a randomized controlled trial," BMC Public Health, vol. 11, article 521, 2011.

[30] B. D. Smedley and S. L. Syme, "Promoting health: intervention strategies from social and behavioral research," American Journal of Health Promotion, vol. 15, no. 3, pp. 149-166, 2001.

[31] N. Pender, C. Murdaugh, and M. Parsons, Health Promotion in Nursing Practice, Prentice Hall, Upper Saddle River, NJ, USA, 2006.

[32] Health Resources and Service Administration, Federal Office of Rural Health Policy (ORHP), and US Department of Agriculture; Economic Research Service, "Rural-urban commuting area codes (RUCAs)," https://depts.washington.edu/uwruca/ index.php.

[33] S. G. Leveille, H. E. Resnick, and J. Balfour, "Gender differences in disability: evidence and underlying reasons," Aging Clinical and Experimental Research, vol. 12, no. 2, pp. 106-112, 2000.

[34] B. J. Cardinal, J. Esters, and M. K. Cardinal, "Evaluation of the revised physical activity readiness questionnaire in older adults," Medicine and Science in Sports and Exercise, vol. 28, no. 4, pp. 468-472, 1996.

[35] C. T. Netzer, The Complete Book of Food Counts, Dell Publishing, New York, NY, USA, 2008.

[36] U.S. Department of Health and Human Services and U.S. Department of Agriculture, Dietary Guidelines for Americans 2010, U.S. Government Printing Office, Washington, DC, USA, 2010.

[37] United States Department of Health \& Human Services, "2008 physical activity guidelines for Americans," Report of the ODPHP Publication, United States Department of Health \& Human Services, Washington, DC, USA, 2008.

[38] U.S. Department of Health and Human Services, "Healthy people 2010: with understanding and improving health," Tech. Rep., U.S. Government Printing Office, Washington, DC, USA, 2000 . 
[39] W. L. Haskell, I.-M. Lee, R. R. Pate et al., "Physical activity and public health: updated recommendation for adults from the American College of Sports Medicine and the American Heart Association," Circulation, vol. 116, no. 9, pp. 1081-1093, 2007.

[40] M. E. Nelson, W. J. Rejeski, S. N. Blair et al., "Physical activity and public health in older adults: recommendation from the American College of Sports Medicine and the American Heart Association," Medicine and Science in Sports and Exercise, vol. 39, no. 8, pp. 1435-1445, 2007.

[41] R. E. Glasgow, S. S. Bull, J. D. Piette, and J. F. Steiner, "Interactive behavior change technology: a partial solution to the competing demands of primary care," American Journal of Preventive Medicine, vol. 27, no. 2 supplement, pp. 80-87, 2004.

[42] United States Department of Health \& Human Services, "Clinical guidelines on the identification, evaluation, and treatment of overweight and obesity in adults-the evidence report," Report of the National Institutes of Health, National Heart Lung and Blood Institute, Bethesda, Md, USA, 1998.

[43] L. S. Boeckner, C. H. Pullen, S. N. Walker, G. W. Abbott, and T. Block, "Use and reliability of the World Wide Web version of the block health habits and history questionnaire with older rural women," Journal of Nutrition Education and Behavior, vol. 34, supplement 1, pp. S20-S24, 2002.

[44] G. Block, M. Woods, A. Potosky, and C. Clifford, "Validation of a self-administered diet history questionnaire using multiple diet records," Journal of Clinical Epidemiology, vol. 43, no. 12, pp. 1327-1335, 1990.

[45] J. A. Mares-Perlman, B. E. K. Klein, R. Klein, L. L. Ritter, M. R. Fisher, and J. L. Freudenheim, "A diet history questionnaire ranks nutrient intakes in middle-aged and older men and women similarly to multiple food records," Journal of Nutrition, vol. 123, no. 3, pp. 489-501, 1993.

[46] P. A. Hageman, C. H. Pullen, M. Hertzog, and L. S. Boeckner, "Effectiveness of tailored lifestyle interventions, using webbased and print-mail, for reducing blood pressure among rural women with prehypertension: main results of the Wellness for Women: DASHing towards Health clinical trial," International Journal of Behavioral Nutrition and Physical Activity, vol. 11, no. 1, article 148, 2014.

[47] R. P. Troiano, D. Berrigan, K. W. Dodd, L. C. Mâsse, T. Tilert, and M. Mcdowell, "Physical activity in the United States measured by accelerometer," Medicine and Science in Sports and Exercise, vol. 40, no. 1, pp. 181-188, 2008.

[48] C. E. Matthews, "Calibration of accelerometer output for adults," Medicine and Science in Sports and Exercise, vol. 37, no. 11 supplement, pp. S512-S522, 2005.

[49] P. S. Freedson, E. Melanson, and J. Sirard, "Calibration of the computer science and applications, inc. accelerometer," Medicine and Science in Sports and Exercise, vol. 30, no. 5, pp. 777-781, 1998.

[50] M. M. Yore, S. A. Ham, B. E. Ainsworth et al., "Reliability and validity of the instrument used in BRFSS to assess physical activity," Medicine and Science in Sports and Exercise, vol. 39, no. 8, pp. 1267-1274, 2007.

[51] J. R. Morrow Jr., T. M. Bain, G. M. Frierson, E. Trudelle-Jackson, and W. L. Haskell, "Long-term tracking of physical activity behaviors in women: The WIN study," Medicine and Science in Sports and Exercise, vol. 43, no. 1, pp. 165-170, 2011.

[52] T. G. Pickering, J. E. Hall, L. J. Appel et al., "Recommendations for blood pressure measurement in humans and experimental animals. Part 1: blood pressure measurement in humans: a statement for professionals from the subcommittee of professional and public education of the American Heart Association council on high blood pressure research," Hypertension, vol. 45, no. 1, pp. 142-161, 2005.

[53] D. B. Sacks, D. E. Bruns, D. E. Goldstein, N. K. Maclaren, J. M. McDonald, and M. Parrott, "Guidelines and recommendations for laboratory analysis in the diagnosis and management of diabetes mellitus," Clinical Chemistry, vol. 48, no. 3, pp. 436$472,2002$.

[54] C. Beunckens, G. Molenberghs, and M. G. Kenward, "Direct likelihood analysis versus simple forms of imputation for missing data in randomized clinical trials," Clinical Trials, vol. 2, no. 5, pp. 379-386, 2005.

[55] C. K. Enders, Applied Missing Data Analysis, Guilford Press, New York, NY, USA, 2010.

[56] M. J. Franz, J. J. VanWormer, A. L. Crain et al., "Weight-loss outcomes: a systematic review and meta-analysis of weight-loss clinical trials with a minimum 1-year follow-up," Journal of the American Dietetic Association, vol. 107, no. 10, pp. 1755-1767, 2007.

[57] J. E. Neter, B. E. Stam, F. J. Kok, D. E. Grobbee, and J. M. Geleijnse, "Influence of weight reduction on blood pressure: a meta-analysis of randomized controlled trials," Hypertension, vol. 42, no. 5, pp. 878-884, 2003.

[58] M. Neve, P. J. Morgan, P. R. Jones, and C. E. Collins, "Effectiveness of web-based interventions in achieving weight loss and weight loss maintenance in overweight and obese adults: a systematic review with meta-analysis," Obesity Reviews, vol. 11, no. 4, pp. 306-321, 2010.

[59] G. Eysenbach, “The law of attrition," Journal of Medical Internet Research, vol. 7, no. 1, article ell, 2005.

[60] I. Moroshko, L. Brennan, and P. O’Brien, "Predictors of dropout in weight loss interventions: a systematic review of the literature," Obesity Reviews, vol. 12, no. 11, pp. 912-934, 2011.

[61] K. L. Funk, V. J. Stevens, L. J. Appel et al., "Associations of internet website use with weight change in a long-term weight loss maintenance program," Journal of Medical Internet Research, vol. 12, no. 3, article e29, 2010.

[62] G. Eysenbach and CONSORT-EHEALTH Group, "CONSORTEHEALTH: improving and standardizing evaluation reports of Web-based and mobile health interventions," Journal of Medical Internet Research, vol. 13, no. 4, article e126, 2011. 


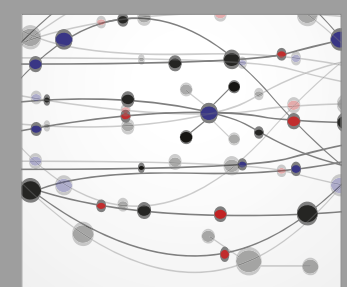

The Scientific World Journal
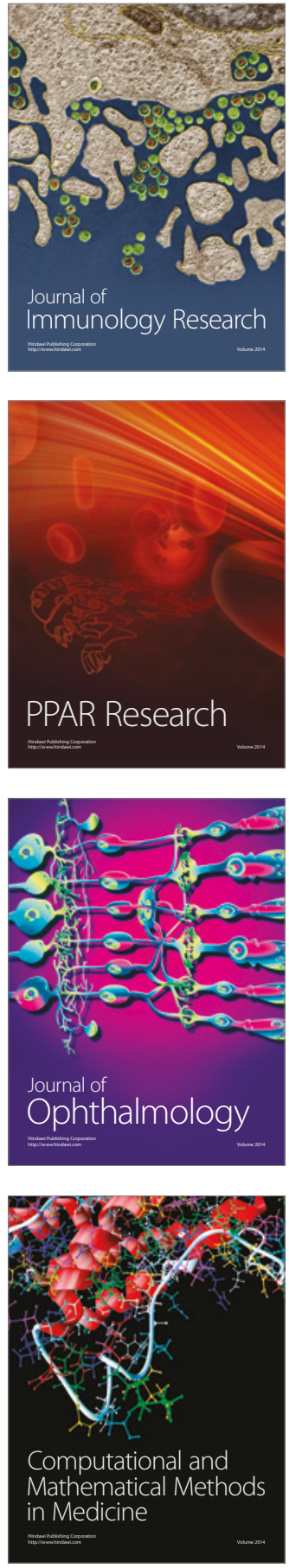

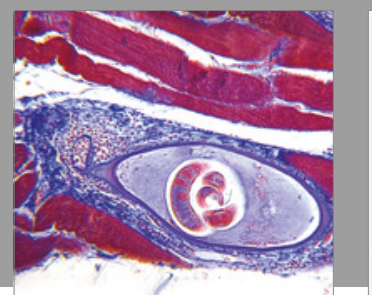

Gastroenterology Research and Practice
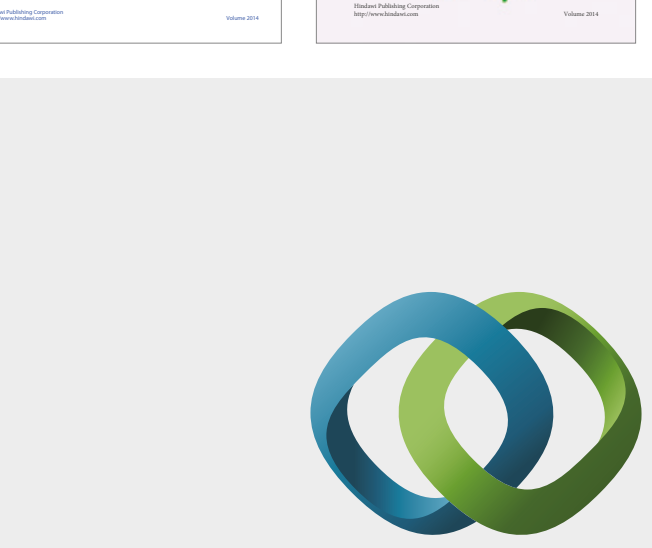

\section{Hindawi}

Submit your manuscripts at

https://www.hindawi.com
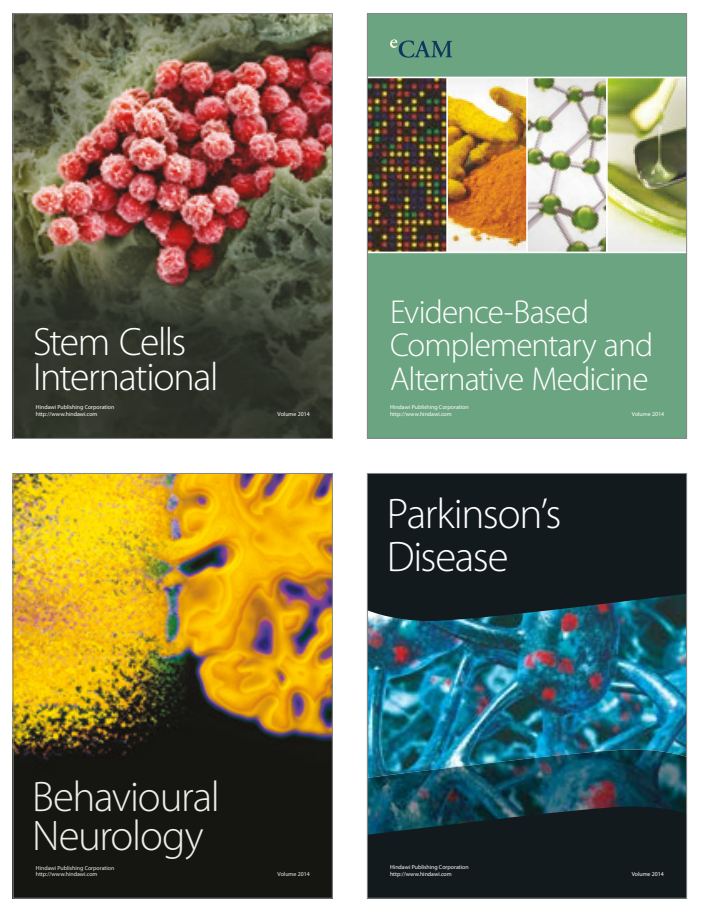
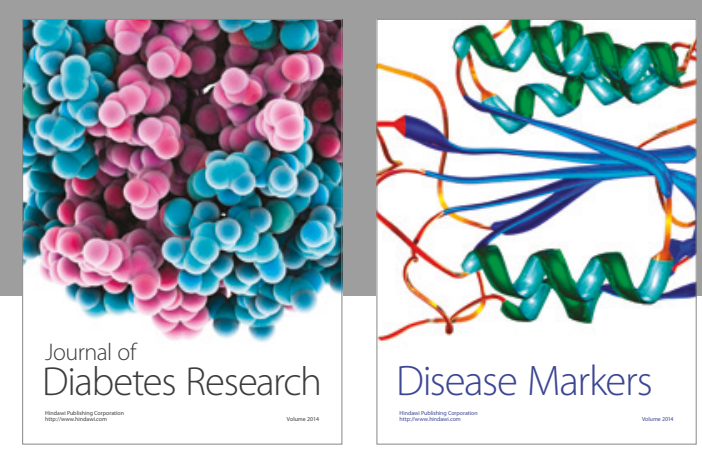

Disease Markers
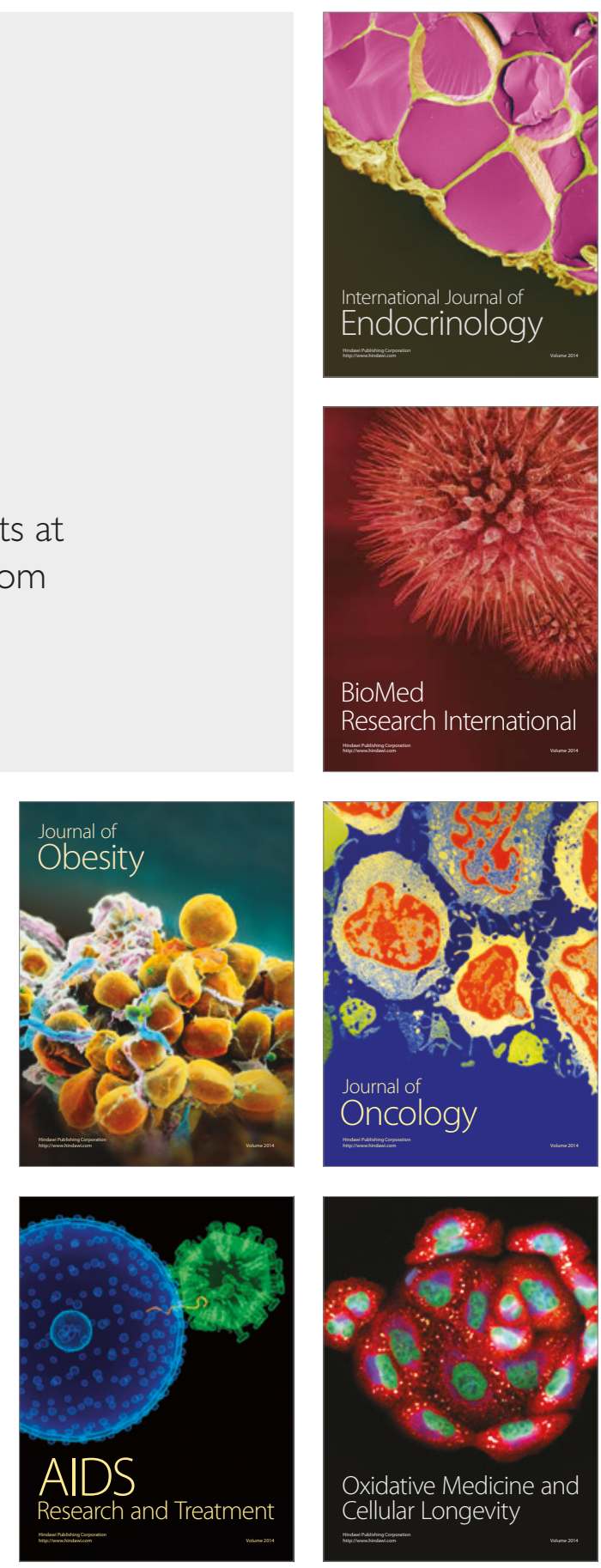